\title{
Inference of Pavement Properties with Roadside Accelerometers
}

\author{
Nielsen, Julius; Levenberg, Eyal; Skar, Asmus
}

Published in:

Proceedings of the 9th International Conference on Maintenance and Rehabilitation of Pavements

Link to article, DOI:

10.1007/978-3-030-48679-2_67

Publication date:

2020

Document Version

Peer reviewed version

Link back to DTU Orbit

Citation (APA):

Nielsen, J., Levenberg, E., \& Skar, A. (2020). Inference of Pavement Properties with Roadside Accelerometers. In Proceedings of the 9th International Conference on Maintenance and Rehabilitation of Pavements (Vol. 76, pp. 719-728). Springer. Lecture Notes in Civil Engineering Vol. 76 https://doi.org/10.1007/978-3-030-48679-2_67

\section{General rights}

Copyright and moral rights for the publications made accessible in the public portal are retained by the authors and/or other copyright owners and it is a condition of accessing publications that users recognise and abide by the legal requirements associated with these rights.

- Users may download and print one copy of any publication from the public portal for the purpose of private study or research.

- You may not further distribute the material or use it for any profit-making activity or commercial gain

- You may freely distribute the URL identifying the publication in the public portal 


\title{
Inference of Pavement Properties with Roadside Accelerometers
}

\author{
Julius Nielsen ${ }^{1}$, Eyal Levenberg ${ }^{1, *}$ and Asmus Skar ${ }^{1}$ \\ ${ }^{1}$ Technical University of Denmark, Department of Civil Engineering, Nordvej, Building 119, \\ 2800 Kgs. Lyngby, \\ eylev@byg.dtu.dk
}

\begin{abstract}
An array of four synchronized single-axis accelerometers was fixed to the surface of an asphalt pavement. Vertical acceleration traces resulting from several nearby passes of a truck with known characteristics were recorded. The work focused on presenting and demonstrating an interpretation method for inferring the mechanical properties of the pavement system based on the recorded accelerations. In general terms, the method was based on carefully low-pass filtering the field-measured acceleration traces, and then best-matching them with a corresponding set of calculated acceleration traces. For this purpose, the pavement system was modeled as a two-layered linear elastic half-space, and a model-guided signal filtering approach was devised to ensure that irrelevant signal content is removed prior to the matching. Based on the analysis of six separate truck passes it was noticed that the inferred upper layer modulus exhibited medium variability while the lower (subgrade) modulus showed little variability. The moduli values displayed fair agreement with those independently estimated from non-destructive and semi-destructive tests. By analyzing many more passes inferred moduli are expected to become more representative. Overall, the method seems workable and scalable, with capacity to handle any number of acceleration sensors as well as other sensor types.
\end{abstract}

Keywords: Pavement condition evaluation; Pavement sensing; Pavement accelerations; Mechanical pavement properties; Model-guided filtering.

\section{Introduction}

Recent years has seen marked increase in public dependency on the service level and overall functionality provided by pavements. This increased dependency is accompanied by user intolerance to service interruptions, part of which are due to activities taken for evaluating mechanical condition. Such condition evaluation data are essential for optimizing maintenance and repair decisions; they are also important for revisiting design decisions, for assessing the performance of non-standard materials or construction technologies, and for estimating loss of structural integrity after natural disasters. Thus the engineering community is challenged to develop non-disruptive technologies for mechanical pavement evaluation. 
One approach for addressing the challenge is building and operating mobile sensing platforms that can carry out condition evaluation while moving alongside the users (Harr and Elton, 1983; Hildebrand and Rasmussen, 2002; Andersen et al., 2017). Another approach, which is the focus of this paper, calls for instrumenting pavements with sensing gear - effectively transforming these traditional systems into smart selfaware constructions (e.g., Lajnef et al., 2011; Klar et al., 2016, Skar et al., 2019). Specifically advocated herein is the use of accelerometers, which of all sensor types, are deemed most suited for installation over wide areas (Ryynäenen et al., 2014; Levenberg et al., 2014).

In the work of Ryynäenen et al. (2014) a low-volume asphalt road, comprised of a thin asphalt layer covering a granular structure, was instrumented with sensors including displacement transducers and near-surface accelerometers. Loading experiments were conducted whereby a truck of known weight and dimensions was driven over the gauge array along different paths and speeds. Measured accelerations were double integrated with respect to time in an attempt to arrive at deflections. It is reported that calculation corrections were necessary (and applied) to offset artificial data drift appearing after the integration. Even so, discrepancy was seen between deflections calculated from accelerations and the measurements of the displacement transducers. A layered elastic pavement model was used to match the deflections and derive layer moduli; the derived moduli, however, seemed unrealistic.

In the work of Arraigada et al. (2009), accelerometers were implanted in two full scale asphalt pavements along with deflectometers. Measured accelerations generated by moving loads were double integrated in an attempt to calculate deflections. Difficulties were reported in arriving at deflections because of the sensitivity of the integration operation to signal noise and drift. Arraigada et al. suggested and applied a spline-based correction in an effort to alleviate the problem. This resulted in 'reasonable qualitative correlation' between direct deflection measurements and deflections calculated from double integrating accelerations. In addition, difficulty was mentioned in dealing with slow-moving loads.

As a means to resolve the issues with double integration of acceleration traces, it was proposed in Levenberg (2012) and in Levenberg (2015) to directly match measured accelerations against computed accelerations. Doing so required, as an added analysis step, the application of a low-pass filter to the measured signals prior to the matching. This filter has the task of removing irrelevant signal content that, if retained, would obstruct the fitting and subsequent inference of properties. A finite impulse response filter type was applied based on a predefined kernel, and the concept of model-guided filtering was introduced for selecting the suitable kernel bandwidth (or effective cutoff frequency). Utilizing synthetic data and field measured data, the approach was demonstrated and successfully applied to infer pavement properties from the readings of a single embedded accelerometer.

The aim of the current contribution is to further advance the idea and techniques for inferring pavement properties based on measured acceleration traces. The overall approach taken is similar to that in in Levenberg (2012) and in Levenberg (2015) where measured signals are not double integrated, but carefully filtered and 
then directly matched against model-generated signals. The new advances being explored herein are: (i) utilizing measurements obtained from an array of synchronized accelerometers and not from a single sensor; (ii) investigating an improved filter concept that is based on truncating a Fourier series and not on a convolution with a smoothing kernel; and (iii) placement of the sensors roadside at the pavement surface and not implanting them below the ride surface. All aforementioned advancement points are closely linked to the goal developing a practical condition evaluation system for in-service pavements based on the readings of roadside sensors.

The paper commences with a description of a field experiment involving an array of four surface mounted accelerometers. Signals were recorded during several nearby passes of a truck having known weight and dimensions. Next, an interpretation method is proposed for assessing pavement properties based on a collection of measured acceleration traces. Then, the method is demonstrated by application to the signal data collected in the field experiment. The paper concludes with a short summary of the work, discussion of the main results, and some ideas for further investigation.

\section{Experimental setup and field measurements}

The experimental investigation was performed in Henrik Dams Allé (Kongens Lyngby, Denmark), which is a single-carriageway straight asphalt road that is $610 \mathrm{~m}$ long. This road has a paved width of $8 \mathrm{~m}$ and includes shallow block-paved drainage ditches on both sides. The pavement structure is $650 \mathrm{~mm}$ thick, composed of $120 \mathrm{~mm}$ asphalt concrete and $530 \mathrm{~mm}$ of combined unbound base and subbase. The subgrade is a relatively stiff sandy clay extending to a large depth. The test section was located in the center of a nominally flat stretch; it was instrumented with four single-axis accelerometers (named Acc\#1...Acc\#4) that were fixed to the asphalt surface via $60 \mathrm{~mm}$ long screws. The sensors were spaced $5 \mathrm{~m}$ apart in the travel direction, with varying transverse offsets from the road centerline: $1.5 \mathrm{~m}$ for Acc\#1 and Acc\#4, $1.7 \mathrm{~m}$ for Acc\#2, and $1.9 \mathrm{~m}$ for Acc\#3. Fig. 1a offers a plan view of the test section, displaying the positions of the sensors. Also shown are three nearby locations where asphalt concrete core samples were obtained for further laboratory investigation and where in situ tests were performed: thickness assessment, dynamic cone penetration testing, and falling weight deflections.

The accelerometers were all identical, model KB12VD manufactured by Metra Mess (Germany). A picture of one such accelerometer attached to the pavement surface is offered in Fig. 1c. The KB12VD sensors are analog, characterized by a measurement range of $\pm 0.6 \mathrm{~g}$ and measurement sensitivity better than $1 \mu \mathrm{g}$. Two interlinked M312 units (also manufactured by Metra Mess) were employed for powering the sensors, data logging, and analog to digital conversion (24 bit). The M312 units were connected to the accelerometers via long cables and also to a laptop via two USB cables for data recording; they offered a synchronized acquisition at a native rate of $96 \mathrm{kHz}$. Given that the KB12VD sensors are not suited for monitoring static or very 
slow-occurring responses, native measurements were preprocessed by high-pass filtering with a cutoff frequency of $1 \mathrm{~Hz}$. Moreover, in order to produce a workable data size for subsequent analysis, and without loss of relevant information, measurements were down-converted to $1 \mathrm{kHz}$ by application of a low-pass filter with $500 \mathrm{~Hz}$ cutoff and subsequent downsampling.

Acceleration responses were generated by the Dynatest Raptor, a heavy truck that hosts a beam of lasers (as well as other sensors) designed to perform pavement evaluation while driving. Fig la superposes a silhouette of the Raptor over the experimental arrangement; the travel speed, assumed constant, is denoted by $V$. Fig $1 \mathrm{~b}$ offers side and top view sketches of the truck, showing tire configuration and indicating axle loads. A right-handed Cartesian coordinate system is introduced with the $x$-axis pointing in the vehicle travel direction, and the $z$-axis points downward into the pavement system, and the $y$-axis pointing transversely to the travel direction with its origin coinciding with the edge of the rear right tire of the track. Assuming the truck was traveling in a straight line parallel to the $x$-axis, the symbol $y_{0}$ is introduced to denote the offset distance from the rear right tire edge to Acc\#1 or equivalently to Acc\#4.

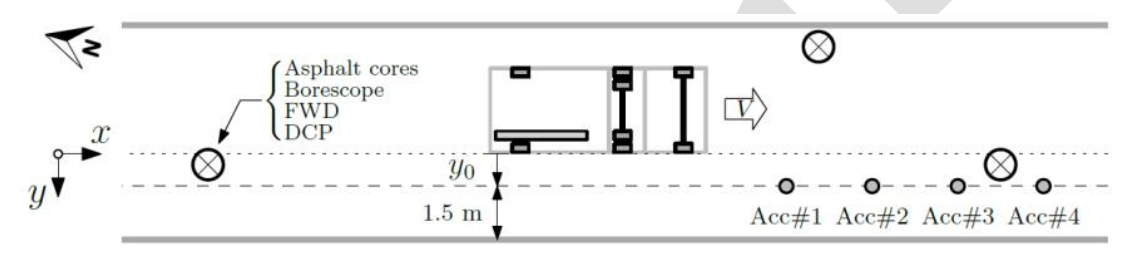

(b)

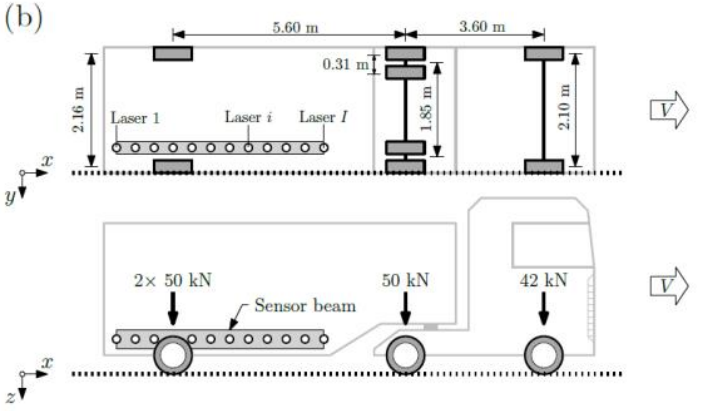

(c)

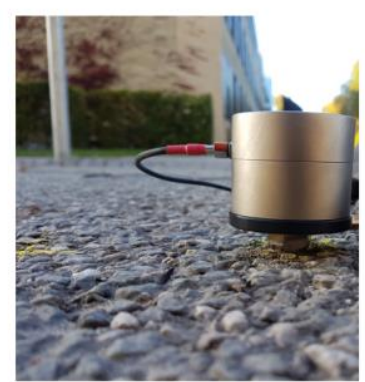

Fig. 1 Experimental setup: (a) top view ( $x-y$ plane) of the test section, (b) sketch of the Raptor truck, and (c) photograph of one accelerometer fixed to the pavement surface.

Testing was done over the course of several hours during which the road was partially closed to traffic. The Raptor truck was instructed to carry out several passes near the sensing array at different speeds. All passes were recorded on video, and the lateral offsets, i.e. the $y_{0}$ 's, were evaluated based on the video footage. To facilitate the offset determination, equidistant lines with $100 \mathrm{~mm}$ spacing were marked on the pavement surface prior to the truck passes. The accuracy level at which lateral offsets were determined with this procedure is estimated to be about $\pm 50 \mathrm{~mm}$. 
Hereafter, data from one of the passes with $y_{0}=250 \mathrm{~mm}$ (Pass $\# 1$ ) are presented and subsequently utilized to demonstrate the interpretation method. This choice is inconsequential because the underlying procedure is essentially generic. The measured accelerations are presented in Fig. 2 with a common timeline. As can be seen, the different sensors recorded the pass with some time lag representing the actual driving speed (Levenberg, 2014). Given the known vehicle dimensions, the truck passing speed was assessed to be $V=57 \mathrm{~km} / \mathrm{h}$. Moreover, is can also be seen that the acceleration intensities vary is accordance with the lateral distance from the truck, e.g., the signal peaks of Acc\#1 and Acc\#4 are relatively similar to one another, while the signal of Acc\#3 seems weakest. As expected (Levenberg, 2015), the lateral offset is seen to have a strong influence on the signal strength; a transverse offset of merely $200 \mathrm{~mm}$ considerably attenuates the acceleration signal (50\% reduced peak).

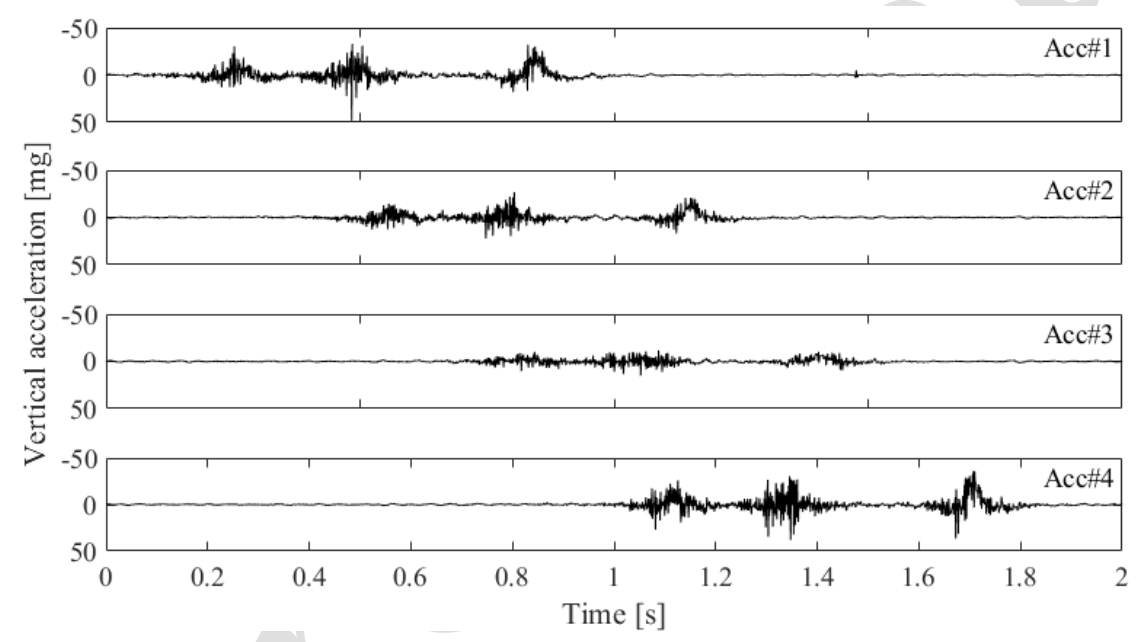

Fig. 2 Measured accelerations in the field experiment for Pass $\# 1\left(y_{0}=250 \mathrm{~mm}\right)$.

\section{Data interpretation method}

The interpretation method described hereafter is focused on assessing the mechanical properties of a tested road based on accelerometer measurements such as those shown in Fig. 2. In general terms, the assessment is sought by best fitting all measured acceleration traces with calculated acceleration traces obtained from a computational pavement model. In this connection, the classic two-layered linear elastic half-space model was chosen (Burmister, 1945), with two unknowns to be inferred: $E_{l}$ - the Young's modulus of the top layer representing the structure, and $E_{2}$ - the Young's modulus of the half-space representing the subgrade. To expedite the underlying computations, the acceleration approach proposed in Andersen et al. (2018) is employed. Taken as 
known in the fitting procedure are: the thickness of the top layer $(650 \mathrm{~mm})$, the Poisson's ratios of the two layers $\left(v_{1}=0.35\right.$, and $\left.v_{2}=0.40\right)$, the travel speed of the truck $(V)$, the truck's tire configuration and axle loads (Fig. 1b), and the lateral offset $y_{0}$ (Fig. 1a).

The fitting procedure is carried out in several steps: (i) for a trial values of $E_{l}$ and $E_{2}$ the layered pavement model is engaged to calculate vertical surface displacement traces $u_{z}(t)$ 's at the sensor locations; (ii) a double derivative with respect to time is applied to the displacement traces to arrive at calculated accelerations - corresponding to the measured signals; (iii) an individual objective function is defined to quantify the matching error between a given measured acceleration trace and it's corresponding calculated trace; (iv) a global objective function is defined, combining all individual objective functions into a scalar entity that represents the matching error between all measured and calculated accelerations; and (v) a search is performed to find optimal moduli that minimize this global objective function. Ultimately, the optimal moduli set is deemed representative of the in situ mechanical pavement layer properties.

The above described procedure requires an additional (intermediate) step prior to defining an individual error function (Levenberg, 2012; 2015). This step entails filtering the measured traces to remove high-frequency signal content that originates from vehicle dynamics and from the interaction between rolling tires and a rough textured surface. To graphically illustrate this requirement, Fig. 3a offers a magnified view of Acc\#4 signal superposed over a corresponding calculated acceleration trace with an assumed moduli set of $E_{1}=300 \mathrm{MPa}$ and $E_{2}=100 \mathrm{MPa}$. As can be seen, the calculated trace is smooth, clearly reflecting the effects of the different truck axles, both in terms of acceleration directions and magnitudes. On the other hand, the measured trace is very noisy, with acceleration levels abruptly changing sign producing a myriad of peaks. Because of this behavior the measured acceleration traces cannot be directly matched against calculated traces. Doing so will result in ill-defined objective functions that cannot facilitate the search for optimal $E_{1}$ and $E_{2}$.
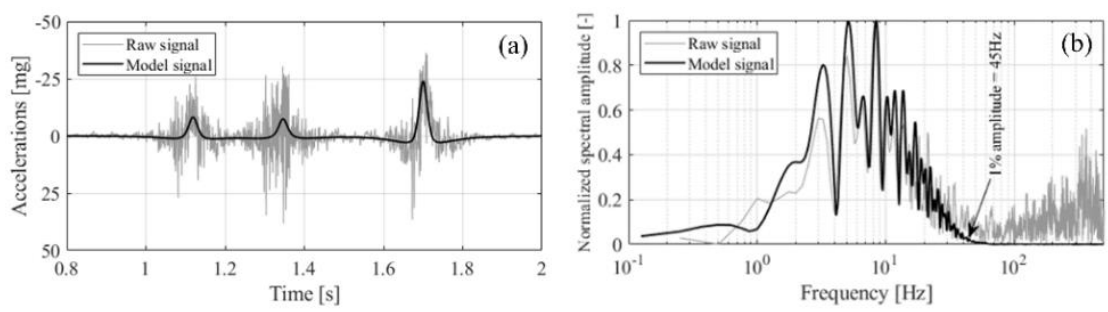

Fig. 3 Synthetic data produced with layer moduli of $E_{l}=300 \mathrm{MPa}$ and $E_{2}=100 \mathrm{MPa}$ compared to raw measurements of Acc\#4: (a) time domain, and (b) frequency domain.

Low-pass filtering of measured traces requires an a priori selection of a certain cutoff frequency. The chosen cutoff is critical for insuring a correct outcome. Choosing a cutoff that is too high retains irrelevant signal content that may contaminate and bias the objective function. On the other hand, choosing a cutoff frequency that is too low removes signal content that holds relevant pavement response information needed for obtaining a correct solution. To resolve this issue, a model-guided 
approach is taken, in which the cutoff frequency is selected based on the frequency content of the calculated acceleration trace that is being used for the matching (Levenberg, 2012, 2015; Drori and Levenberg, 2016). To illustrate this point, Fig. $3 b$ presents the spectral amplitudes of both the measured and calculated acceleration traces of Fig 3a. These spectral magnitudes are normalized by their corresponding peaks and are therefore unitless - ranging between zero and unity. As can be seen, the calculated acceleration trace is essentially band-limited with an effective high frequency of about $45 \mathrm{~Hz}$. This frequency level corresponds to a normalized spectral amplitude of approximately one percent; it essentially means that a Fourier series with frequencies up to $45 \mathrm{~Hz}$ can reproduce the calculated acceleration signal almost perfectly. On the other hand, and as can also be seen in Fig. 3b, the field-measured acceleration signal involves frequencies that are much higher than $45 \mathrm{~Hz}$. Thus, when matching these two traces for evaluating their individual objective function, the measured acceleration signal should first undergo low-pass filtering with a cutoff frequency of $45 \mathrm{~Hz}$. This cutoff is simply applied by first representing the measured signal for the entire duration of the truck passing event as a Fourier series, and then reconstructing the series while excluding all terms associated with frequencies higher than $45 \mathrm{~Hz}$. This procedure is equivalent to the application of a near-perfect filter.

\section{Analysis of properties}

The interpretation method outlined in the previous section is applied herein to infer the layer properties of the tested pavement. The starting point is choosing a trial pair of layer moduli $E_{1}$ and $E_{2}$. Next, acceleration traces are calculated for each of the four sensors; these are concurrently investigated in the frequency domain to obtain a suitable set of four different cutoff frequencies based on the one percent rule - refer to Fig. $3 \mathrm{~b}$. Then, the cutoffs are applied to filter the measured signals - after which four individual objective functions are defined:

$$
\varphi_{j}=\frac{1}{N} \sum_{n=1}^{N}\left|a_{j, n}^{\mathrm{mf}}-a_{j, n}^{\mathrm{c}}\right| \quad(n \in \square)
$$

where $\varphi_{j}=\varphi_{j}\left(E_{1}, E_{2}\right)$ is the individual objective function for Acc\#j, $a_{j, n}^{\mathrm{mf}}$ is the measured and filtered acceleration signal of Acc $\# j$ at time $t_{n}, a_{j, n}^{\mathrm{c}}$ is the corresponding calculated (model-simulated) signal for Acc\# $j$ at time $t_{n}$, and $N$ is the total number of data points utilized for the matching (based on $1 \mathrm{kHz}$ sampling rate). As a means of simultaneously considering the signals from all four sensors, the following so-called min-max multicriterion formulation is employed (Osyczka, 1978; Levenberg, 2013):

$$
\Phi=\sum_{j=1}^{4}\left(\frac{\varphi_{j}}{\varphi_{j}^{0}}-1\right)
$$


wherein $\Phi=\Phi\left(E_{1}, E_{2}\right)$ is a global objective function, $\varphi_{j}$ is the individual objective function defined in Eq. (1), and $\varphi_{j}^{0}$ is the smallest value the objective function $\varphi_{j}$ can attain when minimized with respect to $E_{1}$ and $E_{2}$

The last analysis step is minimizing the global objective function with respect to $E_{1}$ and $E_{2}$ to arrive at the sought pavement properties. This can be attempted with a nonlinear search algorithm (e.g. Lagarias et al., 1998). However, nonlinear search algorithms cannot guarantee converges to a global minimum, especially if the search path is not smooth or contains local minima (or both). Another option, although more computationally demanding, is to densely grid the solution space and simply pick out the global minimum. Shown in Fig. 4a is the outcome of such an approach, corresponding to the acceleration traces in Fig. 2 (i.e., Pass\#1). It provides a contour plot of $\Phi$ for different combinations of $E_{1}$ in the range $50 \ldots 500 \mathrm{MPa}$ and $E_{2}$ in the range $10 \ldots 200 \mathrm{MPa}$. This plot is composed of more than 2000 function evaluations with 10 $\mathrm{MPa}$ intervals for $E_{1}$ and $5 \mathrm{MPa}$ intervals for $E_{2}$. Once a region encapsulating the global minimum was identified, with values in the range $380 \ldots 400 \mathrm{MPa}$ for $E_{l}$ and $80 \ldots 90 \mathrm{MPa}$ for $E_{2}$, the solution grid was further refined (in this region only) to intervals of $1 \mathrm{MPa}$ for of both $E_{1}$ and $E_{2}$; the final (globally optimal) solution is $E_{1}=390$ $\mathrm{MPa}$ and $E_{l}=83 \mathrm{MPa}$. Fig. $4 \mathrm{~b}$ provides two sections of the objective function surface taken through this optimal point. As can be graphically deduced, the behavior of $\Phi$ in the moduli space can be challenging for a nonlinear search algorithm, given the tortuous behavior and existence of local minima. This unfavorable behavior can be ascribed, at least in part, to the fact that the method is based on digital signals, and that the frequency cutoff changes when different trial set of moduli values are assessed.
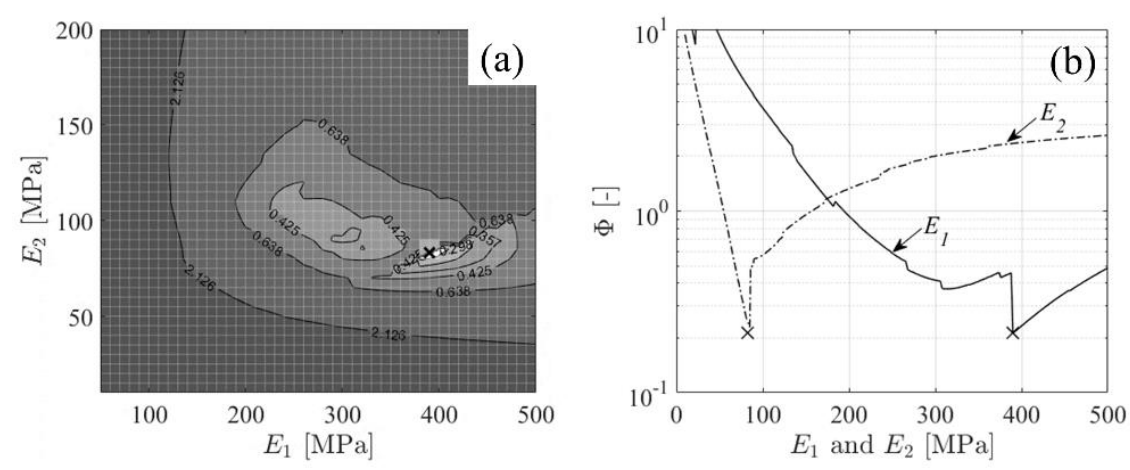

Fig. 4 Plot of the global objective function in Eq. (2) for Pass\#1: (a) contour plot of $\Phi$ as a function of $E_{1}$ and $E_{2}$, and (b) sections through the minimum point (denoted with a cross marker).

Fig. 5 shows the optimal match in the time domain for Pass\#1, superposing calculated acceleration traces (with $E_{1}=390 \mathrm{MPa}$ and $E_{2}=83 \mathrm{MPa}$ ) over their corresponding measured and filtered signals. As can be seen, the match is fairly good across all four sensors. Table 1 presents results from six different passes, listing the lateral offset (from video analysis), the travel speed of the truck, the inferred moduli assuming a two-layered half-space, and the minimal (optimal) value of the corresponding global objective function. To arrive at this table the entire interpretation method was 
essentially repeated. As can be observed, the top layer modulus $\left(E_{l}\right)$ varies within a wide range $163 \ldots 548 \mathrm{MPa}$ while the lower layer (half-space) modulus $\left(E_{2}\right)$ varies within a narrow range $83 \ldots 104 \mathrm{MPa}$.
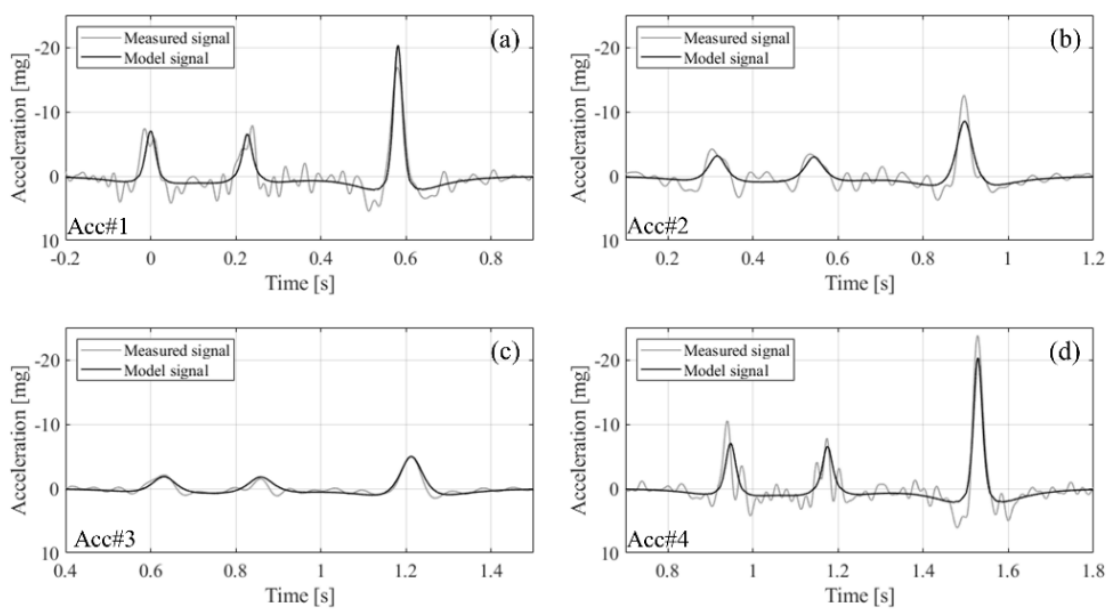

Fig. 5 Optimal match obtained for Pass\#1 between measured (and filtered) accelerations and calculated accelerations in a two-layered half-space model. The time origin $t=0$ denotes when the front axle of the truck is closest to Acc\#1.

Table 1 Layer moduli of the test pavement inferred from roadside accelerometer readings during different truck passes.

\begin{tabular}{cccccc}
\hline Pass \# & $y_{0}[\mathrm{~mm}]$ & $V[\mathrm{~km} / \mathrm{h}]$ & $E_{1}[\mathrm{MPa}]$ & $E_{2}[\mathrm{MPa}]$ & $\Phi$ \\
\hline 1 & 250 & 57 & 390 & 83 & 0.2126 \\
2 & 300 & 35 & 189 & 104 & 0.3086 \\
3 & 300 & 38 & 267 & 91 & 0.2020 \\
4 & 400 & 58 & 347 & 92 & 0.2913 \\
5 & 500 & 23 & 163 & 94 & 0.3698 \\
6 & 400 & 38 & 548 & 87 & 0.4777 \\
\hline
\end{tabular}

\section{Summary and discussion}

An array of four roadside accelerometers was utilized in a field experiment to record several nearby passes of a truck. Assuming a two-layered pavement model, an interpretation method was proposed and applied to infer moduli corresponding to in situ values. The method was based on filtering and then best-matching measured acceleration traces with calculated traces (see Fig. 5). A model-guided approach was employed in the filtering to choose an appropriate cutoff frequency (see Fig. 3). Moduli 
inferred from different passes showed variability (see Table 1): upper layer moduli exhibited medium variability while the lower (subgrade) moduli showed little variability. These variabilities seemed unrelated to: lateral offsets, travel speeds, and optimal (minimal) value of the respective global error functions.

Combining the results from the six different passes into a single moduli set, utilizing a weighted average based on $\Phi^{-1}$, gives: $E_{l}=310 \mathrm{MPa}$ and $E_{2}=91 \mathrm{MPa}$. These values do not compare well with elastostatic backcalculation of falling weight defelectometer results, which gave an upper pavement modulus of $640 \mathrm{MPa}$ and a lower (subgrade) modulus of $170 \mathrm{MPa}$. However, they do compare very well with dynamic cone penetrometer results (Chen et al., 2005), which gave an upper pavement modulus of $340 \mathrm{MPa}$ and a lower (subgrade) modulus of $120 \mathrm{MPa}$. By analyzing many passes and subsequently combining the individual results, inferred moduli are expected to become more representative. Other aspects that may influence the variability and inaccuracy of the interpretation results include: (i) incorrect assessment of the lateral offset $y_{0}$ (see Figure 1); (ii) trucks not driving perfectly parallel to the road centerline; (iii) truck not driving at constant speed while passing next to the sensor array; and (iii) imposition of an improper pavement model on the measurements.

Overall, the proposed method seems to yield a workable and rational solution that avoids the difficulties associated with double integration of acceleration signals. The method also seems scalable, with capacity to handle any number of acceleration sensors as well as other sensor types. Based on the findings of this study, some future research directions are identified: (i) incorporate, as part of the sensing setup, a technology for measuring lateral offsets of passing vehicles; (ii) experiment with more sensors and more sensor types; (iii) include more sophisticated pavement modeling, e.g., more than two layers, edge effects, and viscoelasticity; (iv) treat the truck travel speed as an additional unknown to be inferred from the setup; (v) investigate efficient techniques for finding the global objective function minimum, especially when more than two unknowns are sought; and (vi) assuming a known and calibrated pavement model, try to infer axle weights from the measurements.

\section{References}

Andersen, S., Levenberg, E., and Andersen, M. B. (2017). Inferring pavement layer properties from a moving measurement platform. Proceedings of the 10th International Conference on the Bearing Capacity of Roads, Railways and Air-fields, Loizos A., Al-Qadi, I.L., and Scarpas, T. (Eds), CRC Press/Taylor \& Francis Group, pp. 675-682.

Andersen, S., Levenberg, E., and Andersen, M. B. (2018). Efficient reevaluation of surface displacements in a layered elastic half-space. International Journal of Pavement Engineering, DOI: $10.1080 / 10298436.2018 .1483502$

Arraigada, M., Partl, M. N., Angelone, S. M., and Martinez, F. (2009). Evaluation of accelerometers to determine pavement deflections under traffic loads. Materials and Structures 42(6), 779-790.

Burmister, D. M. (1945). The general theory of stresses and displacements in layered systems. Journal of applied physics 16(2), 89-94 (Part I), 126-127 (Part II), 296-302 (Part III). 
Chen, D. H., Lin, D. F., Liau, P. H., and Bilyeu, J. (2005). A correlation between dynamic cone penetrometer values and pavement layer moduli. Geotechnical Testing Journal 28(1), 42 49.

Drori, O. and Levenberg, E. (2016). Characterization of a traveling object with an underground cluster of accelerometers. Transforming the Future of Infrastructure through Smarter Information - Proceedings of the International Conference on Smart Infrastructure and Construction, ICSIC 2016, 349-356.

Harr, M. E. and Elton, D. J. (1983). Non-contact, non-destructive airport pavement profile, texture and deflection measurements. Report DOT/FAA/PM-83/14, U.S. Department of Transportation, Federal Aviation Administration.

Hildebrand, G. and S. Rasmussen. Development of a high-speed deflectograph. Danish Road Institute, Report 117, Road Directorate, Roskilde, Denmark, 2002.

Klar, A., Levenberg, E., Tur, M., and Zadok, A. (2016). Sensing for smart infrastructure: Prospective engineering applications. Transforming the Future of Infrastructure through Smarter Information - Proceedings of the International Conference on Smart Infrastructure and Construction, ICSIC 2016, 289-295.

Lagarias, J. C., Reeds, J. A., Wright, M. H., and Wright, P. E. (1998). Convergence properties of the Nelder-Mead simplex method in low dimensions. SIAM Journal on optimization 9(1), $112-147$.

Lajnef, N., Rhimi, M., Chatti, K., Mhamdi, L., and Faridazar, F. (2011). Toward an integrated smart sensing system and data interpretation techniques for pavement fatigue monitoring. Computer-aided Civil and Infrastructure Engineering 26(7), 513-523.

Levenberg, E. (2012). Inferring pavement properties using an embedded accelerometer. International Journal of Transportation Science and Technology 1(3), 229-246.

Levenberg, E. (2013). Inverse analysis of viscoelastic pavement properties using data from embedded instrumentation. International Journal for Numerical and Analytical Methods in Geomechanics 37(9), 1016-1033

Levenberg, E. (2014). Estimating vehicle speed with embedded inertial sensors. Transportation Research Part C: Emerging Technologies 46, 300-308.

Levenberg, E. (2015). Backcalculation with an implanted inertial sensor. Transportation Research Record 2525, 3-12.

Levenberg, E., Shmuel, I., Orbach, M., and Mizrachi, B. (2014). Wireless pavement sensors for wide-area instrumentation. Sustainability, Eco-efficiency and Conservation in Transportation Infrastructure Asset Management - Proceedings of the 3rd International Conference on Transportation Infrastructure, ICTI 2014, 307-319.

Osyczka, A. (1978). Approach to multicriterion optimization problems for engineering design. Computer Methods in Applied Mechanics and Engineering 15(3), 309-333.

Ryynänen, T., Pellinen, T., and Belt, J. (2014). The use of accelerometers in the pavement performance monitoring and analysis. IOP Conference Series: Materials Science and Engineering 10(1), 012110.

Skar, A., Klar, A., and Levenberg, E. (2019). Load-independent characterization of plate foundation support using high-resolution distributed fiber-optic sensing. Sensors 19(16), 3518 